\title{
Highly Stable Surface Functionalization of Microgas Chromatography Columns Using Layer-by-Layer Self-Assembly of Silica Nanoparticles
}

\author{
Dong Wang, ${ }^{\dagger}$ Hamza Shakeel, ${ }^{\ddagger}$ John Lovette, ${ }^{\S}$ Gary W. Rice, ${ }^{\S}$ James R. Heflin, ${ }^{\dagger}$ and Masoud Agah*, ${ }^{\ddagger}$ \\ ${ }^{\dagger}$ Department of Physics, Virginia Tech, Blacksburg, Virginia 24061, United States \\ ${ }^{*}$ VT MEMS Lab Bradley Department of Electrical \& Computer Engineering, Virginia Tech, Blacksburg, Virginia 24061, United States \\ ${ }^{\S}$ Department of Chemistry, The College of William \& Mary, Williamsburg, Virginia 23187, United States
}

ABSTRACT: A controllable and high-yield surface functionalization of silicon microchannels using layer-by-layer (LbL) self-assembly of $\mathrm{SiO}_{2}$ nanoparticles (SNPs) is presented. The application of SNPs (45 nm average diameter) coating as a stationary phase for chromatographic separation is also demonstrated with surface functionalization using chloroalkylsilanes. This method facilitates a simple, low-cost, and parallel processing scheme that also provides homogeneous and stable nanoparticle-based stationary phases with ease of control over the coating thickness. The SNP-functionalized microfabricated columns with either single capillary channels ( $1 \mathrm{~m}$ long, 150 $\mu \mathrm{m}$ wide, $240 \mu \mathrm{m}$ deep) or very narrow multicapillary channels

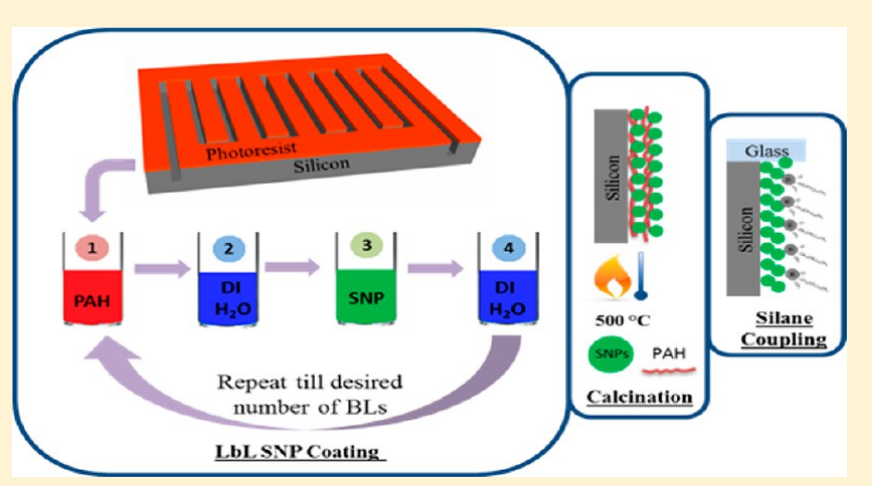
( $25 \mathrm{~cm}$ long, $30 \mu \mathrm{m}$ wide, $240 \mu \mathrm{m}$ deep, 16 parallel channels) successfully separated a multicomponent gas mixture with a wide range of boiling points with high reproducibility.

$\mathrm{B}$ ecause of recent advancements and the emergence of microelectromechanical systems (MEMS), energy-efficient integrated microgas chromatography $(\mu \mathrm{GC})$ systems have attracted considerable attention. This system, upon complete realization, could expand the range of applications for real-time and rapid on-site analysis at a lower cost. ${ }^{1-10}$ As a key component in $\mu \mathrm{GC}$ systems, conventional meters-long separation columns are necessarily replaced with microfabricated silicon columns on the order of a few square centimeters. The separation ability and efficiency of the columns are directly related to the quality of the stationary phase. Using conventional coating techniques on microfabricated columns (e.g., dynamic or static coatings of functionalized polysiloxane polymers), especially for highaspect-ratio (HAR) rectangular-shaped capillary channels, present major challenges toward obtaining stable, reproducible, and uniform coatings. ${ }^{11}$

There have been progressive research efforts toward the development of coating techniques that yield chemically inert, thermally stable, selective, and robust stationary phases. Specifically, nanotechnology-based phases have opened up countless prospects for applications in conventional as well as microfabricated separation columns with nanoscale controllability, simplicity, and flexibility. ${ }^{12,13}$ Recently, carbon nanotubes $^{14,15}$ and thiol-encapsulated gold nanoparticles ${ }^{16}$ have been utilized for gas chromatography (GC) separations. Our group has reported on the capability of thiol monolayers on electrodeposited gold surfaces as a stationary phase for microfabricated columns by combining nanofabrication and microfabrication techniques. ${ }^{17,18}$

The excellent adsorption properties of silica for organics has long been demonstrated as a stationary phase medium for conventional columns (silica gel, Celite) ${ }^{19}$ and, more recently, by employing the MEMS-compatible sputtering technique for microfabricated columns. ${ }^{20} \mathrm{SiO}_{2}$ nanoparticles (SNPs), because of their small and uniform size, high surface area, chemical inertness, and thermal stability, are excellent candidates for stationary phases. SNPs have also been dispersed separately in ethanol and ionic liquid (IL) solutions for conventional capillary columns using static coating methods. ${ }^{21}$ The SNPsIL phase was reported to offer superior separation capability, because of complete coverage of the inner capillary wall surface.

Developed first in $1991,{ }^{22,23}$ the LbL method was used to fabricate ionic self-assembled multilayers thin films, which have been used in a wide range of applications including electromechanical actuators, ${ }^{24-28}$ chemical and biological sensors, $^{29-35}$ and optical ${ }^{36-40}$ and electrochromic devices. ${ }^{41-47}$ In short, by alternately immersing the substrate into two aqueous solutions of oppositely charged materials, LbL thin films of the two materials are electrostatically bound together and can be built up to very large numbers of multilayers (a pair of such layers is referred to as one bilayer). Thorough rinsing

Received: April 12, 2013

Accepted: July 27, 2013

Published: July 27, 2013 


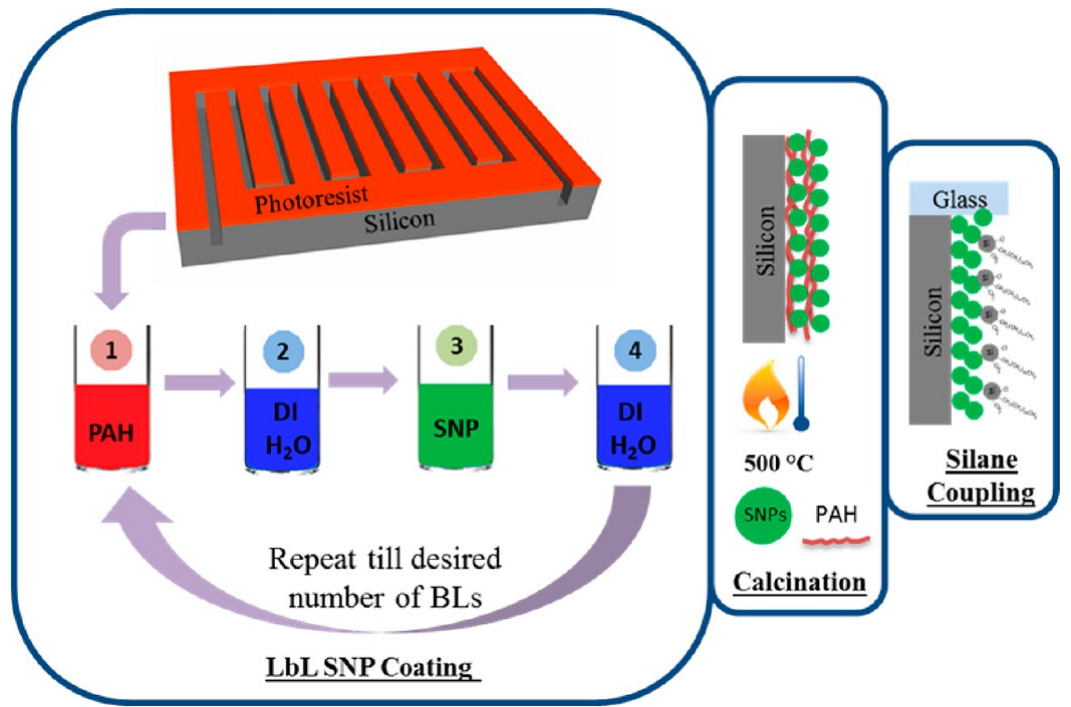

Figure 1. Schematic process flow of SNPs coating using layer-by-layer self-assembly technique in the silicon channel.

between each deposition step washes away excess material from the previous stage while leaving enough surface charge for the next material to be electrostatically deposited. Thus, uniform, dense, and robust stacks of layers can be deposited on the surface of the substrate with a wide variety of configurations. A desired film thickness can be achieved by simply terminating the procedure after a certain number of bilayers (BLs). Carbon nanotubes, ${ }^{48,49}$ gold nanoparticles, ${ }^{50}$ and SNPs ${ }^{51,52}$ have been deposited via LbL assembly on flat as well as etched microfluidic surfaces ${ }^{53,54}$ for diverse nanotechnology-driven applications.

We present here the utilization of SNP-based stationary phases for $\mu \mathrm{GC}$ columns using LbL self-assembly that affords homogeneous and controllable film thickness. Both 1-m-long micro-single-capillary columns ( $\mu \mathrm{SCC})$ and 25 -cm-long micromulticapillary columns ( $\mu \mathrm{MCC})$ can be coated with ease. It is important to note that $\mu \mathrm{MCC}$ with very narrow widths and parallel channels provide high sample capacity, high separation efficiencies, and rapid analysis times. ${ }^{55,56}$ Moreover, with the relatively simple concept and straightforward process, LbL assembly could be conveniently incorporated into the parallel manufacturing of silicon-based $\mu \mathrm{GC}$ devices.

\section{MATERIALS AND REAGENTS}

Singled-side polished silicon wafers ( 4 in., $500 \mu$ m thick, $n$ type) and double-sided polished (4 in., $500 \mu \mathrm{m}$ thick) Pyrex wafers used for sealing the microchannels were purchased from University Wafers. Fused-silica capillary tubing $(220 \mu \mathrm{m}$ outer diameter, $100 \mu \mathrm{m}$ inner diameter, Polymicro Technologies) was used for the microfluidic interface. SNPs suspension (45 $\mathrm{nm}$ average diameter, $20-21 \mathrm{wt} \%$ in water) was purchased from Nissan Chemical. All chemicals including those used for chromatographic testing were of HPLC standard grade and purchased directly from Sigma-Aldrich. Ultrapure gases (>99.99\%) including nitrogen and air were obtained from Airgas.

\section{FABRICATION}

The fabrication scheme incorporating LbL self-assembled SNPs schematically shown in Figure 1 can be divided into four major steps, which include column fabrication, LBL deposition of
SNPs, calcination and anodic bonding of the silicon substrate with Pyrex, followed by deactivation of the silica surface.

Column Fabrication. The fabrication of HAR 1-m-long, $150-\mu \mathrm{m}$-wide, $240-\mu \mathrm{m}$-deep $\mu$ SCC and 25 -cm-long, $30-\mu \mathrm{m}$ wide, $240-\mu \mathrm{m}$-deep $\mu \mathrm{MCC}$ with an array of 16 channels working in parallel start with anisotropic etching of plain silicon wafers using standard MEMS processes. In the first step, wafers are spin-coated with AZ9260 photoresist at $3000 \mathrm{rpm}$, softbaked at $110{ }^{\circ} \mathrm{C}$ for $1 \mathrm{~min}$ and exposed for $45 \mathrm{~s}$ using a mask aligner (Karl Suss MA-6). After development using AZ400K, wafers are hard-baked at $110{ }^{\circ} \mathrm{C}$ for $2 \mathrm{~min}$. Deep reactive-ion etching (DRIE, Alcatel) with photoresist as the etch mask is used to etch the wafers in order to get the desired channel dimensions. Afterward, the wafer is diced into individual devices. Selective deposition of nanoparticles inside the channels is achieved by using a liftoff procedure explained in the next section.

LbL Deposition of SNPs. Keeping the photoresist intact, the LbL deposition of SNPs inside the HAR microfluidic channels starts with alternately dipping each device into a positively charged long-chain polymeric aqueous solution (10 $\mathrm{mM}$ polyallylamine hydrochloride, $\mathrm{PAH}$ ), and then the negatively charged SNPs suspension. Because of the very narrow channels, the $\mu \mathrm{MCC}$ column deposition was performed using the SNPs suspension at one-third of the original concentration ( $7 \mathrm{wt} \%$ ) to prevent the formation of bulky nanoparticle structures between the channel walls. Following our earlier reported findings, ${ }^{57}$ the $\mathrm{pH}$ value of the PAH solution and SNPs suspension were adjusted to $7.0( \pm 0.1)$ and $9.0( \pm 0.1)$, respectively, by adding $\mathrm{HCl}$ and $\mathrm{NaOH}$ solutions to guarantee excellent $\mathrm{PAH} / \mathrm{SNPs} \mathrm{LbL}$ coating uniformity. The LbL coating is performed in an automatic dipping system (StratoSequence VI Robot, nanoStrata, Inc.) in which every 2.5 min coating step with PAH and SNPs is followed by three 1min rinsing steps with deionized water (DI) water (see Figure 1). The attractive electrostatic interactions between negatively charged SNPs and positively charged PAH layer results in the formation of each bilayer. The LbL deposition scheme presented here affords homogeneous SNPs coverage both on the interior microchannel surfaces and on the photoresist at the top of chip. After coating, the samples are rinsed thoroughly with DI water and dried with low flux nitrogen. After 
deposition of the desired number of bilayers, the devices are then dipped in acetone and sonicated for $12 \mathrm{~min}$. This liftoff procedure removes the SNPs deposited on the photoresist on top of the channel walls without removing the coating inside the channels. This is especially important for microfluidic applications in which the SNPs coated silicon channels are to be sealed with a glass substrate.

Calcination. Before anodic bonding with the Pyrex cover plate, the devices are first calcinated (Figure 1) in a furnace at a high temperature $\left(500{ }^{\circ} \mathrm{C}\right)$ for $4 \mathrm{~h}$, to slightly fuse the SNPs together to ensure the stability of the coating during temperature programming of the GC columns while keeping the spherical shape of the SNPs intact. It has been reported that calcination of SNPs at higher temperatures can eliminate the binding polymer $(\mathrm{PAH})$ and/or fuse SNPs together, ${ }^{58}$ resulting in a packed homogeneous film.

Anodic bonding and silane-coupling. After calcination, SNP-coated silicon columns are sealed to a Pyrex wafer using anodic bonding at $400{ }^{\circ} \mathrm{C}$ and $1250 \mathrm{~V}$. Deactivated capillary tubes are then installed at the inlet and outlet ports using epoxy. The presence of active silanol (hydroxyl) groups on the surface of silica and SNPs has been widely reported in the literature and are known to be detrimental to chromatographic performance. Deactivation of these groups using chlorosilanes has been shown to reduce peak broadening considerably. In this study, a $10 \mathrm{mM}$ chlorodimethyloctadecyl silane (CDOS) solution in toluene was pumped into the chip by nitrogen (Figure 1) and allowed to sit overnight to deactivate the nanoparticles and glass surface. ${ }^{8}$ The solvent was then removed by nitrogen prior to using the device for chromatographic analysis.

\section{CHARACTERIZATION OF SNP COATING}

To validate the simplicity, repeatability, and robustness of the proposed stationary phase deposition technique, different HAR $\mu \mathrm{GC}$ column configurations ( $\mu \mathrm{SCC}$ and $\mu \mathrm{MCC}-16$ ) were coated using SNPs. The effect of the SNP coating thickness on chromatographic separations was also evaluated by coating $\mu$ SCC devices with 5, 10, and 15 BLs, respectively. The SEM micrographs (Figures $2 \mathrm{~A}-\mathrm{F}$ ) for the devices after calcination clearly show that the bottom and sidewalls of the trenches of $\mu$ SCC are completely covered by SNPs of uniform film thickness. Moreover, the lift-off procedure successfully removed the SNPs from the top surface. The controllability of film thickness using the $\mathrm{LbL}$ approach is demonstrated by SEM micrographs of 5,10 , and 15 bilayers (Figures $2 \mathrm{G}, 2 \mathrm{~F}$, and $2 \mathrm{H}$ ) deposited inside the silicon trenches, respectively. The thickness range measured on the surface inside the channel directly from the SEM at different locations were $\sim 260-320$ $\mathrm{nm}, \sim 400-490 \mathrm{~nm}$, and $\sim 560-610 \mathrm{~nm}$ for 5,10 , and 15 bilayers, respectively. The range of each thickness (as high as a $20 \%$ deviation from the average thickness) is somewhat broader than what is typical for a commercial GC capillary coating $(<5 \%)$. Nevertheless, this clearly demonstrates that film thicknesses are proportional to the number of coating steps and that the combination of microlithography and LbL selfassembly for selective SNPs deposition on the interior surfaces of $\mu \mathrm{GC}$ columns can readily be achieved.

In addition to wide channel $(150 \mu \mathrm{m}) \mu \mathrm{SCC}$, the $\mathrm{LbL}$ coating method was used on very narrow width channels (20 $\mu \mathrm{m})$ inside $\mu \mathrm{MCC}$ configurations. It was observed that the original concentration used for $\mu$ SCC (20-21 wt \% in water) resulted in bulky nanoparticle "bridges" between the sidewalls

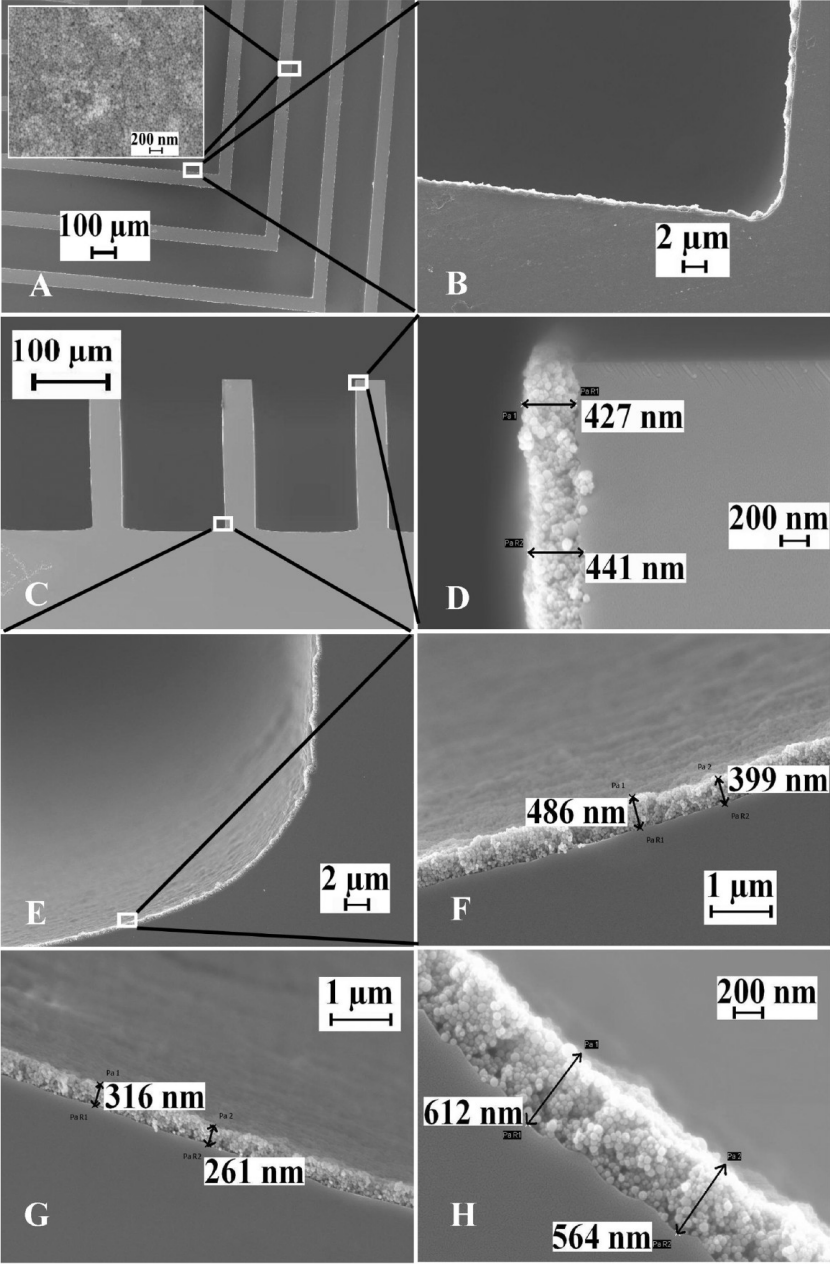

Figure 2. SEM images of the 10-BL SNP-coated $\mu$ SCC (top view and inset), showing (A) the coating on the bottom of the channel, (B) the coating on the sidewall as viewed from top, $(\mathrm{C}-\mathrm{F})$ cross-sectional view of channel with 10-BL SNPs on the inner surfaces, (D) top of the sidewall, (E and F) bottom corner; and 10- (F), (G) 5-, and (H) 15BL SNPs coating with thickness value.

due to the narrow channel widths in $\mu \mathrm{MCC}$, resulting in channel clogging (Figure 3C). The nanoparticle bridges were eliminated by reducing the original concentration of the SNPs suspension (Figure 3D) to $7 \mathrm{wt} \%$ relative to that used for the $\mu$ SCC. Although a lower concentration of SNPs suspension was used, the 10 bilayers coating inside the microfluidic channels was approximately the same thickness $(380 \mathrm{~nm}$, Figure $3 \mathrm{~B})$ as the 10 bilayers coating inside the $\mu \mathrm{SCC}$ (Figure $2 \mathrm{~F}$ ). This implies that the coating in SNPs suspensions at a lower concentration is sufficient to deposit a layer of SNPs over the previous $\mathrm{PAH}$ layer to neutralize and reverse the surface charge, while the excess SNPs during the $\mu$ SCC coating with the higher concentration suspension are most likely washed off during the rinsing steps.

\section{RESULTS AND DISCUSSION}

A commercial GC system (HP 5890 Series II) equipped with an autosampler, electronic pressure controller, and flame ionization detector (FID) was used to characterize the separation ability of the $\mu \mathrm{GC}$ columns coated with the SNP stationary phase. Highly purified nitrogen was used as the carrier gas. Inlet and detector temperatures were maintained at 


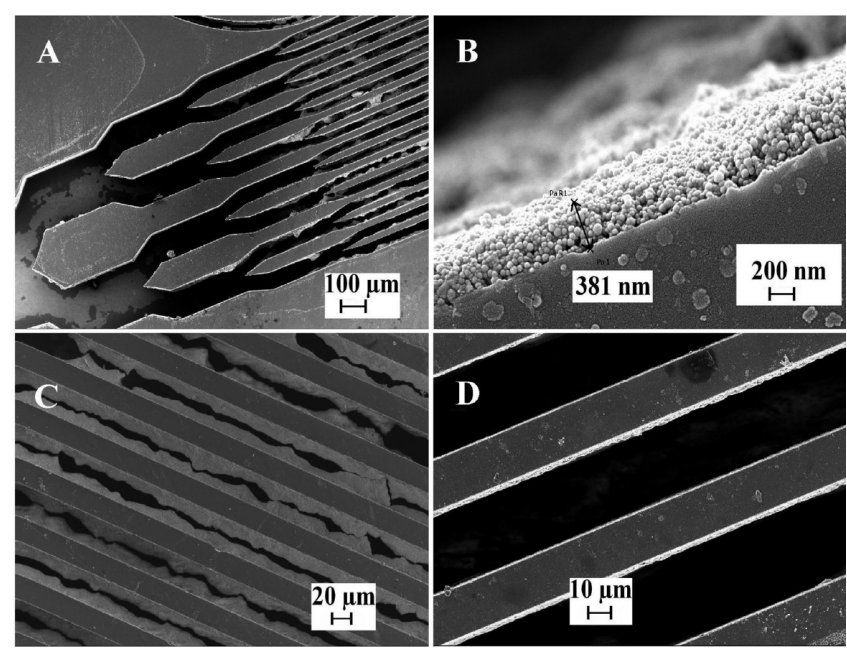

Figure 3. SEM image of (A) the inlet and 16 parallel channels of $\mu \mathrm{MCC}$, and (B) its cross-section with 10 BLs SNPs coating on the sidewall of silicon channel with thickness value. Also shown are coatings on the sidewall of multicapillary $\mu \mathrm{GC}$ column using SNPs suspension with (C) the original concentration and (D)a concentration of 7 wt \%.

$280{ }^{\circ} \mathrm{C}$ with a split ratio set to $100: 1$. In order to evaluate the chromatographic performance, the functionalized $\mu \mathrm{GC}$ columns were placed in a conventional GC oven. All of the columns were first purged with dry nitrogen to remove any trapped oxygen in the system. Columns were conditioned gradually from ambient temperature to slightly above the maximum operating temperature $\left(250{ }^{\circ} \mathrm{C}\right)$ of the column at a rate of $1{ }^{\circ} \mathrm{C} / \mathrm{min}$ and a constant carrier gas flow of 7.5 psi until a constant baseline was observed. The maximum operating temperature of our microfabricated devices is simply limited by the epoxy seals currently used for the capillary connections.

Silane Deactivation. The improvement in the separation performance from CDOS deactivation is illustrated in Figure 4, where a mixture of $\mathrm{C}_{10}, \mathrm{C}_{11}$, and $\mathrm{C}_{12}$ was used to test the $\mu \mathrm{SCC}$

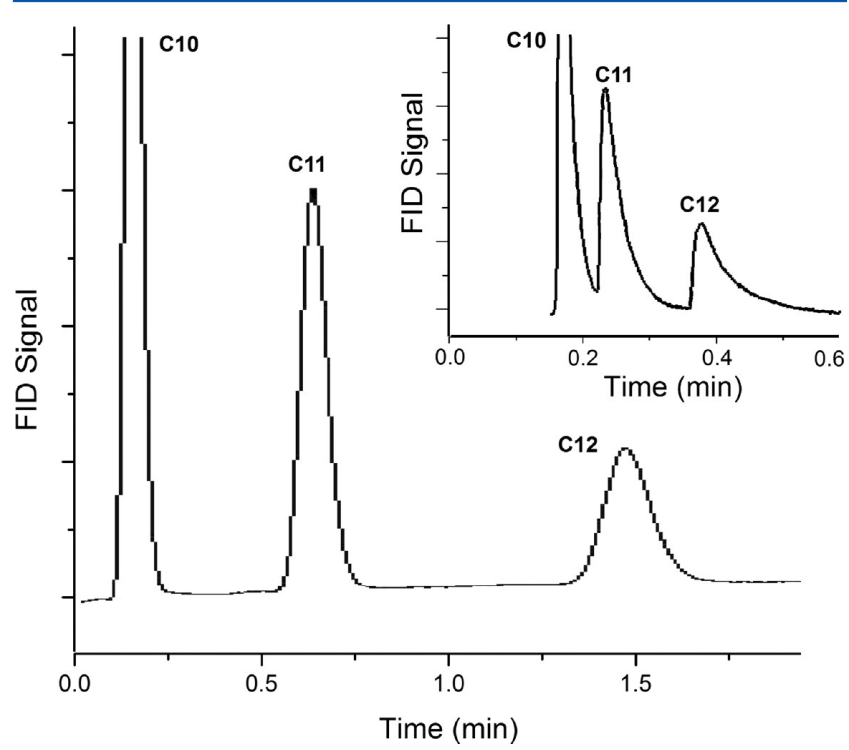

Figure 4. Improvement in separation performance after silane deactivation of 10-BL $\mu \mathrm{SCC}$; inset shows column without silane treatment. Chromatographic conditions: 10 psi with 100:1 split injection ratio and isothermal temperature $=80^{\circ} \mathrm{C}$. before and after silane coupling. The $\mu \mathrm{GC}$ column treated with silane clearly generated sharper and more-symmetric peaks. CDOS was specifically chosen for the deactivation since the chlorinated end group readily reacts with active silanol groups on the $\mathrm{SiO}_{2}$ surface to produce a covalent bond. The long nonpolar octadecyl chain can also enhance the column performance through interactions with the compounds being separated. Improvements in both chromatographic peak symmetry and baseline are also observed for columns subjected to the calcination process.

Separation Results. As a first step, the coating thickness for the reported $\mu \mathrm{SCC}$ was optimized using peak tailing/ symmetry as a figure of merit. As discussed earlier, columns coated with 5, 10, and 15 BLs of nanoparticles provide different phase thicknesses (Figure 2). All three columns were able to successfully resolve alkane test mixtures. Table 1 illustrates

Table 1. Comparison of Retention Times for Three Alkanes for 5-, 10-, and 15-BL SNP-Coated $\mu$ SCCs, and the Same 10BL $\mu$ SCC after Conditioning

\begin{tabular}{lccc} 
& \multicolumn{3}{c}{ Retention Times (min) } \\
\cline { 2 - 4 } 5 BLs & $\mathrm{C}_{10}$ & $\mathrm{C}_{12}$ & $\mathrm{C}_{14}$ \\
$10 \mathrm{BLs}$ & 0.01 & 0.05 & 0.92 \\
$15 \mathrm{BLs}$ & 0.07 & 0.46 & 1.48 \\
second 10 BLs & 0.11 & 0.68 & 1.65 \\
conditioned 10 BLs & 0.08 & 0.49 & 1.55 \\
& 0.05 & 0.30 & 1.40 \\
\hline
\end{tabular}

retention times for three alkanes on each device. As expected, the thickest phase (15 BLs) provided the highest retention times. However, 5- and 15-BL coated columns showed highly unsymmetric peaks, while the $\mu$ SCC with $10 \mathrm{BLs}$ had highly symmetric peaks. Additional characterizations from this point for deviations due to variations in fabrication processes, coating stability, and separation capabilities were made with columns coated with 10-BL SNPs. Deviations in fabrication processes were first considered, with $\sim 10 \%$ variation in retention times observed for $\mu \mathrm{SCC}$ columns produced on two different wafers and coated with 10 BLs of SNPs (Table 1). The separation results of both devices closely resembled each other and had excellent peak symmetry. Similarly, the long-term stability of the SNPs coating under GC testing conditions was monitored by subjecting a $10-\mathrm{BL}$ coated $\mu \mathrm{SCC}$ to a conditioning process (constant flow at $7.5 \mathrm{psi}$ at $160{ }^{\circ} \mathrm{C}$ for $24 \mathrm{~h}$ ). The SNP-based coating was found to have a good stable baseline after this accelerated testing with only modest differences in peak quality and retention times. This demonstrates the ability to produce stable, consistent, and robust stationary phases with small deviations in column performance.

The separation capability of SNPs coating with 10 BLs for $\mu \mathrm{SCC}$ is successfully demonstrated using a series of alkanes (Figure 5), and a 10-compound standard test mixture (Figure 6) containing a variety of commonly found volatile organic compounds (VOCs) to evaluate the resolving power of the SNPs columns. The chromatograms obtained from these columns had excellent reproducibility from multiple injections.

In order to validate our proposed scheme using narrow channel dimensions, the LbL-fabricated SNP coating was also used to functionalize a $\mu \mathrm{MCC}$ with modified SNP concentrations as previously described. Figure 7 shows a chromatogram obtained from the alkane test mix using a 10-BL SNPcoated 16-channel $\mu \mathrm{MCC}$ that was also silane-treated. The 


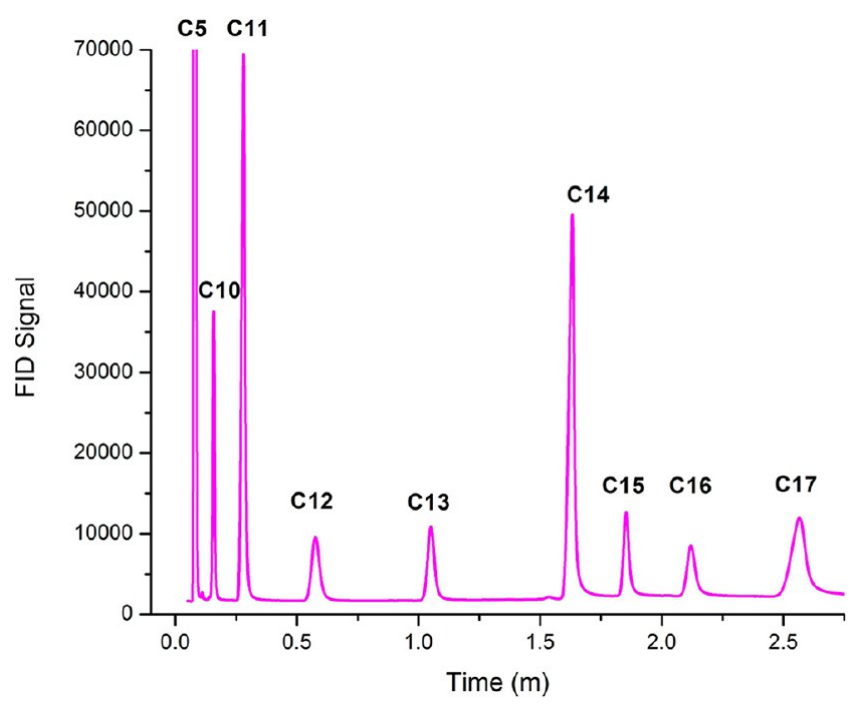

Figure 5. Separation of nine straight chain alkanes using a 10-BL SNP $\mu$ SCC. Chromatographic conditions: 10 psi with 100:1 split injection ratio and temperature program $\left(50{ }^{\circ} \mathrm{C}-120{ }^{\circ} \mathrm{C}\right.$ at a rate of $70{ }^{\circ} \mathrm{C} /$ $\min )$.

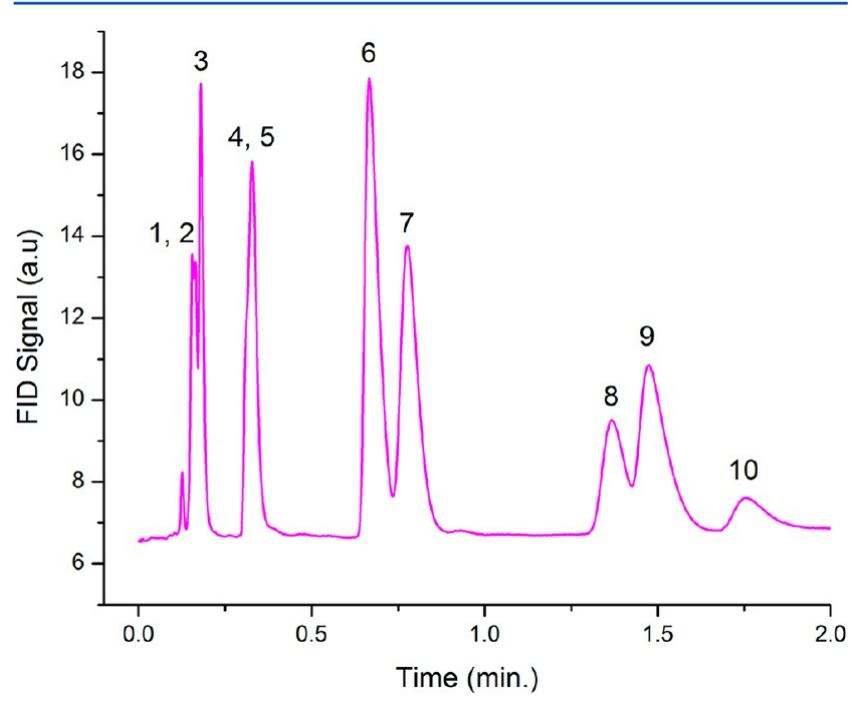

Figure 6. Separation of a 10-component volatile organic compound (VOC) mixture using a $\mu$ SCC 10-BL SNP column. Chromatographic conditions: 10 psi with a 10:1 split injection ratio and a temperature program $\left(30-50{ }^{\circ} \mathrm{C}\right.$ at $\left.10{ }^{\circ} \mathrm{C} / \mathrm{min}\right)$. Compound identification: 1 , dichloromethane; 2, chloroform; 3 , carbon tetrachloride; 4, dibromomethane; 5, tetrachloroethylene; 6, toluene; 7, chlorobenzene; 8 , bromobenzene; 9, $p$-xylene; and 10, 1,1,2,2-tetrachloroethane.

reason for the shift in baseline has not been determined, but it is clear that retention times are markedly longer and potentially provide for greater separating capabilities. These results also indicate that these newly developed SNPs coating schemes should be applicable to other channel geometries and column configurations.

The figure of merit commonly used for column performance is height equivalent to a theoretical plate (HETP),

$$
\text { HETP }=\frac{L}{N}
$$

where $L$ is the length of the column and $N$ is the number of plates in the column, as determined experimentally from peak retention times $\left(t_{\mathrm{r}}\right)$ and widths at half the peak height $\left(w_{1 / 2}\right)$ :

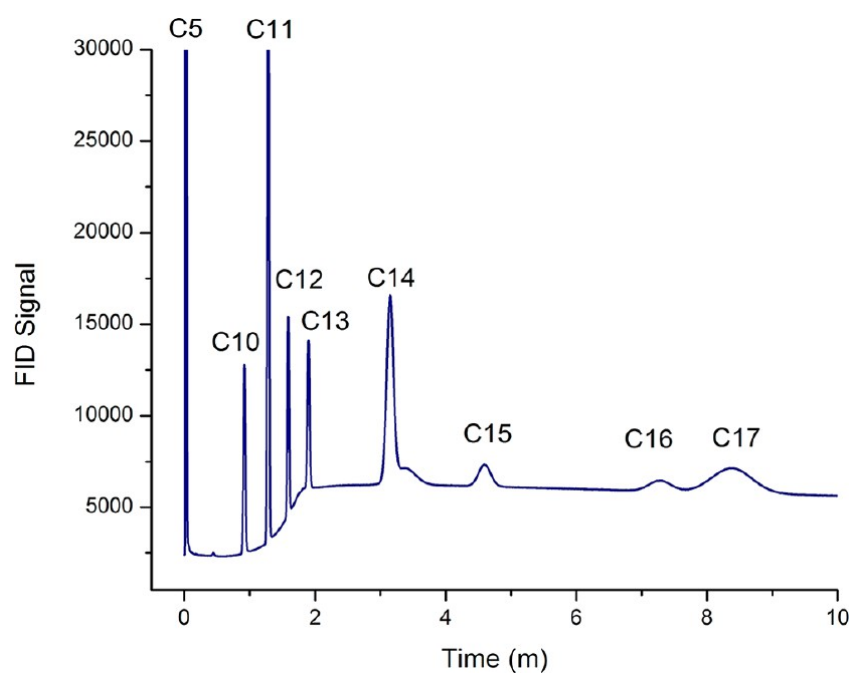

Figure 7. Separation performance of a 16-channel $\mu \mathrm{MCC}$ functionalized with 10-BL SNPs using the alkane test mixture. Chromatographic conditions: 10 psi with 100:1 split injection ratio and a temperature program $\left(50{ }^{\circ} \mathrm{C}-120^{\circ} \mathrm{C}\right.$ at a rate of $\left.70{ }^{\circ} \mathrm{C} / \mathrm{min}\right)$.

$$
N=\left(\frac{t_{r}}{w_{1 / 2}}\right)^{2}
$$

To evaluate the efficiency of the SNP-functionalized $\mu \mathrm{GC}$ columns, the theoretical plate numbers were obtained over a range of column pressures using dodecane diluted in dichloromethane at $65{ }^{\circ} \mathrm{C}$. The maximum plate numbers for the 1 -m-long $\mu \mathrm{SCC}$ and $25-\mathrm{cm}$-long $\mu \mathrm{MCC}$ columns were experimentally calculated to be $930 / \mathrm{m}$ and $4750 / \mathrm{m}$, respectively. The much-larger plate number for the multicapillary column is best explained by the narrower channels, which enhances interactions with the analytes and improves the overall capacity of the column. Plots showing the correlation of the inlet pressure to gas velocity and HETP versus gas velocity are shown in Figure 8. The linear velocity of the mobile phase was estimated from correlating the inlet pressure to the retention time obtained from methane injections, which was assumed to be essentially unretained. In addition to the much lower HETP values, the narrower channel $\mu \mathrm{MCC}$ provide relatively flat Golay plots over a broader range of flow rates, compared to $\mu$ SCC.

The static coating of SNPs in ethanol suspension, reported earlier for capillary columns, ${ }^{21}$ resulted in agglomeration of nanoparticles affording a nonuniform film. The effect of this nonuniformity was also demonstrated in poor chromatographic performance. In the same article, the authors integrated ILs with SNPs to develop a uniform stationary phase $(0.4-0.6 \mu \mathrm{m})$ with improved separation characteristics. Comparatively, our proposed 10-BL SNP coating scheme with a similar film thickness $(0.4-0.5 \mu \mathrm{m})$ enables a homogeneous, stable, controllable, and conformal stationary phase bed. Moreover, by incorporating calcination and silane deactivation, the chromatographic performance (especially peak symmetry) of our LbL technique is superior, compared to reported static coating methods. ${ }^{21}$

\section{CONCLUSION}

Homogeneous $\mathrm{SiO}_{2}$ nanoparticle (SNP) coatings of highly controlled thickness fabricated by the layer-by-layer (LbL) 


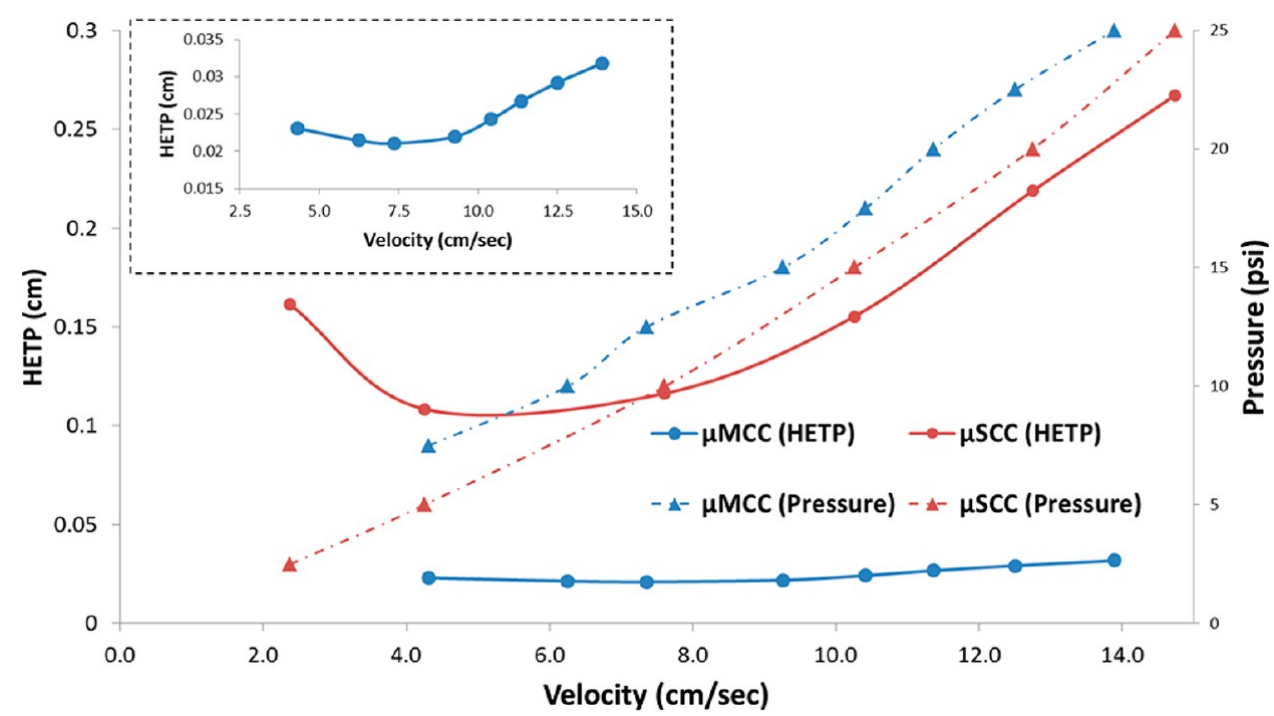

Figure 8. HETP-gas velocity-pressure plots for $\mu$ SCC and $\mu \mathrm{MCC}$ with a 10-BL SNP coating. The dashed lines with triangle symbols are plots of inlet pressure against gas velocity, using methane vapor as an unretained marker, and the solid lines with circle symbols are Golay plots (HETP versus gas velocity). Inset represents the $\mu \mathrm{MCC}$ results on an expanded scale.

technique have been incorporated into microgas chromatography $(\mu \mathrm{GC})$ separation columns and utilized as a stationary phase. With simple and parallel fabrication procedures, the LbL technique has shown the ability to generate a homogeneous conformal SNP coating with good nanoscale thickness control inside the $\mu \mathrm{GC}$ columns with different configurations. The $\mu \mathrm{GC}$ columns (both regular and multicapillary) with this stationary phase show good separation for alkanes with a wide range of boiling points with excellent consistency and repeatability. A more-complex standard test mixture with 10 volatile organic compounds (VOCs) was also successfully separated. This production scheme results in high yield and can be easily integrated into standard MEMS-based fabrication processes. Moreover, the combination of unique properties of SNPs and facile coating procedures could also be applied to conventional GC columns. The next logical steps are to determine methods to reduce variations in the coating thicknesses, as well as study the effect of the size and shape of the SNPs on the chromatographic separations coupled with various chlorosilane functionalities.

\section{AUTHOR INFORMATION}

\section{Corresponding Author}

*E-mail: agah@vt.edu.

\section{Author Contributions}

D.W. and H.S. contributed equally.

\section{Notes}

The authors declare no competing financial interest.

\section{ACKNOWLEDGMENTS}

This work is supported primarily by the National Science Foundation (NSF), under Award No. ECCS-1002279. The authors gratefully acknowledge the support from Mr. Donald Leber during fabrication at MicrON clean room at Virginia Tech. SEM micrographs were taken at the Nanoscale Characterization and Fabrication Laboratory, Institute for Critical Technology and Applied Science, Virginia Tech.

\section{REFERENCES}

(1) Reddy, K.; Guo, Y.; Liu, J.; Lee, W.; Khaing Oo, M. K.; Fan, X. Lab Chip 2012, 12, 901-905.

(2) Jian, R.-S.; Huang, R.-X.; Lu, C.-J. Talanta 2012, 88, 160-167.

(3) Shakeel, H.; Rice, G.; Agah, M. First reconfigurable MEMS separation columns for micro gas chromatography. In 25th IEEE International Conference on Micro Electro Mechanical Systems (MEMS), Paris, France, 2012; pp 823-826.

(4) Narayanan, S.; Alfeeli, B.; Agah, M. IEEE Sens. J. 2012, 12, 18931900.

(5) Serrano, G.; Paul, D.; Kim, S.-J.; Kurabayashi, K.; Zellers, E. T. Anal. Chem. 2012, 84, 6973-6980.

(6) Potkay, J. A.; Lambertus, G. R.; Sacks, R. D.; Wise, K. D. J. Microelectromech. Syst. 2007, 16, 1071-1079.

(7) Lewis, P. R.; Manginell, R. P.; Adkins, D. R.; Kottenstette, R. J.; Wheeler, D. R.; Sokolowski, S. S.; Trudell, D. E.; Byrnes, J. E.; Okandan, M.; Bauer, J. M.; Manley, R. G.; Frye-Mason, C. IEEE Sens. J. 2006, 6, 784-795.

(8) Radadia, A. D.; Masel, R. I.; Shannon, M. A.; Jerrell, J. P.; Cadwallader, K. R. Anal. Chem. 2008, 80, 4087-4094.

(9) Manginell, R.; Bauer, J.; Moorman, M.; Sanchez, L.; Anderson, J.; Whiting, J.; Porter, D.; Copic, D.; Achyuthan, K. Sensors 2011, 11, $6517-6532$

(10) Kaanta, B. C.; Chen, H.; Zhang, X. J. Micromech. Microeng. 2010, 20, 055016.

(11) Zareian-Jahromi, M. A.; Ashraf-Khorassani, M.; Taylor, L. T.; Agah, M. J. Microelectromech. Syst. 2009, 18, 28-37.

(12) Mittermuller, M.; Volmer, D. A. Analyst 2012, 137, 3195-3201.

(13) Guihen, E. TrAC, Trends Anal. Chem. 2013, 46, 1-14.

(14) Yuan, L.-M.; Ren, C.-X.; LiLi, Ai, P.; Yan, Z.-H.; Zi, M.; Li, Z.-Y. Anal. Chem. 2006, 78, 6384-6390.

(15) Herrera-Herrera, A. V.; González-Curbelo, M. Á.; HernándezBorges, J.; Rodríguez-Delgado, M. Á. Anal. Chim. Acta 2012, 734, 130.

(16) Gross, G. M.; Nelson, D. A.; Grate, J. W.; Synovec, R. E. Anal. Chem. 2003, 75, 4558-4564.

(17) Zareian-Jahromi, M. A.; Agah, M. J. Microelectromech. Syst. 2010, 19, 294-304.

(18) Shakeel, H.; Agah, M. J. Microelectromech. Syst. 2013, 22, 62-70.

(19) Bartle, K. D.; Myers, P. TrAC, Trends Anal. Chem. 2002, 21, $547-557$.

(20) Vial, J.; Thiébaut, D.; Marty, F.; Guibal, P.; Haudebourg, R.; Nachef, K.; Danaie, K.; Bourlon, B. J. Chromatogr. A 2011, 1218, $3262-3266$. 
(21) Na, N.; Cui, X.; De Beer, T.; Liu, T.; Tang, T.; Sajid, M.; Ouyang, J. J. Chromatogr. A 2011, 1218, 4552-4558.

(22) Decher, G.; Hong, J. D. Makromol. Chem., Macromol. Symp. 1991, 46, 321-327.

(23) Decher, G.; Hong, J. D.; Schmitt, J. Thin Solid Films 1992, 210, 831-835.

(24) Zeng, T.; Claus, R.; Zhang, F.; Du, W.; Cooper, K. L. Smart Mater. Struct. 2001, 10, 780-785.

(25) Montazami, R; Liu, S.; Liu, Y.; Wang, D.; Zhang, Q.; Heflin, J. R. J. Appl. Phys. 2011, 109, 104301.

(26) Fukushima, T.; Asaka, K.; Kosaka, A.; Aida, T. Angew. Chem., Int. Ed. 2005, 44, 2410-2413.

(27) Liu, S.; Montazami, R.; Liu, Y.; Jain, V.; Lin, M.; Zhou, X.; Heflin, J. R.; Zhang, Q. M. Sens. Actuators, A 2010, 157, 267-275.

(28) Raoufi, N.; Surre, F.; Sun, T.; Rajarajan, M.; Grattan, K. T. V. Sens. Actuators, B 2012, 169, 374-381.

(29) Kim, J. H.; Kim, S. H.; Shiratori, S. Sens. Actuators, B 2004, 102, 241-247.

(30) Wang, B. Z.; Du, X. Y.; Wang, M. Q.; Gong, W. L.; Anzai, J. Electroanalysis 2008, 20, 1028-1031.

(31) Evtugyn, G. A.; Hianik, T. Curr. Anal. Chem. 2011, 7, 8-34.

(32) Sun, P.; Jiang, Y. D.; Xie, G. Z.; Du, X. S.; Li, X.; Hu, J. Sci. China Inf. 2011, 54, 2680-2686.

(33) Cui, L. L.; Pu, T.; Liu, Y.; He, X. Q. Electrochim. Acta 2013, 88, 559-564.

(34) Del Villar, I.; Matias, I. R.; Arregui, F. J.; Lalanne, P. Opt. Express 2005, 13, 56-69.

(35) Wang, Z.; Heflin, J. R.; Van Cott, K.; Stolen, R. H.; Ramachandran, S.; Ghalmi, S. Sens. Actuators, B 2009, 139, 618-623.

(36) Marletta, A.; Castro, F. A.; Borges, C. A. M.; Oliveira, O. N.; Faria, R. M.; Guimaraes, F. E. G. Macromolecules 2002, 35, 91059109.

(37) Yu, H. H.; Jiang, D. S.; Li, X. F.; Yu, D. S.; Zhou, L. D. In Advanced Materials and Devices for Sensing and Imaging II; Wang, A., Zhang, Y., Ishii, Y., Eds.; International Society for Optical Engineering (SPIE): Bellingham, WA, 2005; pp 470-473.

(38) Wang, S.; Zhao, L.; Zhang, X.; Shi, Z.; Cui, Z.; Yang, Y. J. Colloid Interface Sci. 2009, 336, 470-476.

(39) Khan, Z. A.; Kumar, R.; Mohammed, W. S.; Hornyak, G. L.; Dutta, J. J. Mater. Sci. 2011, 46, 6877-6882.

(40) Heflin, J. R.; Guzy, M. T.; Neyman, P. J.; Gaskins, K. J.; Brands, C.; Wang, Z.; Gibson, H. W.; Davis, R. M.; Van Cott, K. E. Langmuir 2006, 22, 5723-5727.

(41) Montazami, R.; Jain, V.; Heflin, J. R. Electrochim. Acta 2010, 56, 990-994.

(42) Jain, V.; Sahoo, R.; Mishra, S. P.; Sinha, J.; Montazami, R.; Yochum, H. M.; Heflin, J. R.; Kumar, A. Macromolecules 2008, 42, 135-140.

(43) Shinbo, K.; Kato, K.; Kaneko, F.; Onishi, K.; Advincula, R. C.; Fan, X. W. Mol. Cryst. Liq. Cryst. 2003, 407, 493-500.

(44) Jain, V.; Khiterer, M.; Montazami, R.; Yochum, H. M.; Shea, K. J.; Heflin, J. R. ACS Appl. Mater. Interfaces 2009, 1, 83-89.

(45) Schmidt, D. J.; Pridgen, E. M.; Hammond, P. T.; Love, J. C. J. Chem. Educ. 2010, 87, 208-211.

(46) Silva, C. H. B.; Galiote, N. A.; Huguenin, F.; Teixeira-Neto, E.; Constantino, V. R. L.; Temperini, M. L. A. J. Mater. Chem. 2012, 22, 14052-14060.

(47) DeLongchamp, D. M.; Hammond, P. T. Chem. Mater. 2004, 16, 4799-4805.

(48) Taylor, A. D.; Michel, M.; Sekol, R. C.; Kizuka, J. M.; Kotov, N. A.; Thompson, L. T. Adv. Funct. Mater. 2008, 18, 3003-3009.

(49) Li, X.; Gittleson, F.; Carmo, M.; Sekol, R. C.; Taylor, A. D. ACS Nano 2012, 6, 1347-1356.

(50) Kong, B.-S.; Geng, J.; Jung, H.-T. Chem. Commun. 2009, 0, 2174-2176.

(51) Wang, F.; Peters, S.; Guzda, J.; Blunk, R. H.; Angelopoulos, A. P. Langmuir 2009, 25, 4384-4392.

(52) Lvov, Y.; Ariga, K.; Onda, M.; Ichinose, I.; Kunitake, T. Langmuir 1997, 13, 6195-6203.
(53) Kim, J. Y.; DeRocher, J. P.; Mao, P.; Han, J.; Cohen, R. E.; Rubner, M. F. Chem. Mater. 2010, 22, 6409-6415.

(54) DeRocher, J. P.; Mao, P.; Han, J.; Rubner, M. F.; Cohen, R. E. Macromolecules 2010, 43, 2430-2437.

(55) Zhdanov, V. P.; Sidelnikov, V. N.; Vlasov, A. A. J. Chromatogr. A 2001, 928, 201-207.

(56) Rosenkranz, B.; Bettmer, J. Anal. Bioanal. Chem. 2002, 373, 461-465.

(57) Yancey, S. E.; Zhong, W.; Heflin, J. R.; Ritter, A. L. J. Appl. Phys. 2006, 99, 034313.

(58) Cebeci, F. Ç.; Wu, Z.; Zhai, L.; Cohen, R. E.; Rubner, M. F. Langmuir 2006, 22, 2856-2862. 